\title{
Personalidad y rendimiento académico en estudiantes universitarios
}

\author{
Isabel Niño de Guzmán ${ }^{1}$, Arturo Calderón ${ }^{2}$ y Mónica Cassaretto ${ }^{3}$ \\ Pontificia Universidad Católica del Perú
}

Se investigó la relación entre personalidad, rendimiento académico y otras variables en 170 universitarios, mediante dos instrumentos validados para esta población: el NEO PI-R de Costa y McCrae y el EPPS de Edwards ${ }^{4}$. Se consideró las variables edad, ciclo académico, percepción del rendimiento, de la propia motivación para estudiar, y fuentes de apoyo. Se confirmo la validez y confiabilidad de ambas pruebas y sus respectivas correlaciones. Se encontraron asociaciones entre el rendimiento y conciencia, perseverancia, cambio y agresión. El rendimiento fue mejor explicado por la aspiraciones de logro, reflexión, cambio, percepción del rendimiento y edad del estudiante.

Palabras clave: rasgos de personalidad, motivación, rendimiento académico, NEO PI-R, EPPS, universitarios.

Personality and academic achievement in university students

The correlations among personality, academic performance and other variables in 170 university students were studied, using two instruments validated into the population: the NEO PI-R, and the EPPS. Other variables as age, study level, self perception of academic achievement and self perception of motivation, and sources of support were included. It was confirmed the reliability and validity of both tests and the correlations between them. The results showed associations among academic performance and conscientiousness, endurance, change, and aggression. The academic performance was better explained by achievement striving, deliberation, change, self perception of academic achievement and age.

Key words: Personality traits, motivation, academic performance, NEO PI-R, EPPS, university students.

1 Magister en Psicología. Profesora auxiliar de la Especialidad de Psicología PUCP.

2 Licenciado en Matemáticas. Docente de la Facultad de Matemáticas PUCP.

$3 \quad$ Licenciada en Psicología Clínica. Profesora de la Especialidad de Psicología.

4 NEO PI-R Inventario de Personalidad NEO de Costa y McCrae (1992) adaptado por Cassaretto (1999). EPPS. inventario de Preferencias Personales de Edwards (1959), traducido por Thorne, C. (1991). 

El papel central de la personalidad en el rendimiento académico solo ha sido puesto de relieve en los últimos años, rompiendo de esta forma el casi exclusivo protagonismo ostentado por la inteligencia (Nácher, 2002). Muchas investigaciones prueban actualmente que el rendimiento académico no sólo se asocia con factores intelectuales, sino que es un efecto de múltiples variables adaptativas, comportamentales y psicopatológicas, a los que se atribuye incluso un valor predictivo (Cascón, 2002). Por ello, se afirma que la inteligencia, personalidad y motivación asociadas entre sí pueden explicar el $25 \%$ de la varianza del rendimiento (Cattell, en Nácher, 2002).

Las personas muestran diferentes formas de aprender, de abordar los contenidos educacionales y de experimentar situaciones académicas. Se ha comprobado que los factores de personalidad determinan gran parte de estas diferencias, pero la mayor parte de los estudios plantean relaciones de correlación entre personalidad y rendimiento académico (Digman, en Nácher, 2002) sin lograr determinar relaciones causa-efecto ni la manera específica en que la personalidad juega un papel determinante, siendo probable que las características generadoras del éxito académico estén en función de la persona, su edad, métodos de enseñanza utilizados, etc.

Muchos trabajos refieren aspectos de la personalidad que afectan al rendimiento; entre ellos se encuentra la extraversión-introversión, neuroticismo, estabilidad emocional, conciencia, motivación por el cambio, entre otros. Entre los rasgos de personalidad más mencionadas se encuentra la extraversión la cual se ha asociado con la realización de proyectos personales (Little, Lecci y Watkinson, 1992), facilidad para la adquisición de información, interés y reforzamiento en las interacciones sociales (afiliación), mayor capacidad de persuasión 
(ascendencia), elevados niveles de actividad, energía y alta dedicación para la obtención de metas (Hogan, Johnson, y Briggs, 1997). En el ámbito escolar se han reportan asociaciones entre extraversión con la evaluación del profesor sobre la adaptación y la conducta académica del alumno (Graciano y Ward, 1992). En el ámbito profesional extraversión se considera un predictor de rendimiento válido para puestos que involucran interacción social, gerencia y ventas Barrick y Mount (1991). Sin embargo, las relaciones entre este rasgo y rendimiento aparecen mediatizada por el sexo siendo positiva para las mujeres en tareas de comunicación verbal, lenguaje, matemática y participación en actividades no académicas. Predominando en varones la relación entre conciencia y rendimiento, logro y participación en actividades que demandan una aproximación y un análisis organizado, práctico y cuidadoso. Esta diferencia disminuye con la edad y la complejidad de las tareas; con los años, los introvertidos se encontrarían en ventaja al centrarse más en tareas escolares; las extravertidas encontrarían en el grupo una mayor aceptación del rendimiento y éxito académico (Entwistle y Cunningham, en Nácher, 2002).

Se encuentra asociación consistente entre conciencia (voluntad de logro), desempeño laboral y logro académico (Digman, 1989). Conciencia propicia el cumplimiento de tareas, competencia intelectual (Gelatly, 1996), metas altas y la convicción de obtenerlas (Cassaretto, 1999), organización diligente y trabajo duro, manejo del ambiente y solución de problemas (Costa y McCrae, 1992). Se relaciona con ambición, seriedad, exactitud, disciplina, método, persistencia (Digman, 1990), destreza y capacidad para el entrenamiento en distintos ambientes. Sujetos con altos niveles en este rasgo se caracterizan por tomarse más tiempo ante las tareas, analizan y proceden cuidadosamente en la resolución de problemas, cometen menos errores que los que responden impulsivamente y al azar (Watson y Lindgren, 1991). Los individuos con baja conciencia son descritos como flojos, descuidados, imprecisos, desorganizados e impetuosos con relación a sus expectativas de desempeño y la elección de metas (Barrick y Mount, 1991). 
En nuestro medio se ha estudiado la relación entre personalidad, tipo de ansiedad e ingreso a la universidad (Niño de Guzmán et al., 2000), los resultados de este estudio señalan correlaciones significativas entre el coeficiente de inteligencia, conciencia e ingreso. Entre las facetas que correlacionan con el ingreso destaca autodisciplina.

Apertura es considerado un predictor importante del logro intelectual. Se asocia al pensamiento divergente, habilidad creativa, facilidad de captación y adquisición de conocimiento, imaginación, perceptividad, y aspectos motivacionales (Costa y McCrae, 1992; Hogan, Johnson, y Briggs, 1997; McCrae y Costa, 1997). Es visto como el sentido de sofisticación, amplitud, profundidad, permeabilidad, interés intelectual y estético frente a la cultura. Las personas con altos puntajes en conciencia y apertura son descritas como intelectualmente curiosas, imaginativas, inventivas, eficientes, organizadas, competentes y cuidadosas en el trabajo (McCrae, 1994).

En el Perú, Claux (1998) ha evidenciado la influencia y capacidad predictiva en el desempeño escolar de los factores apertura, conciencia, y extroversión. Así, encontró que apertura se relacionaba positivamente con desempeño escolar en pruebas de lenguaje y en la participación en actividades no académicas. El factor conciencia se encontraba asociado con el logro en pruebas de matemáticas y la participación en actividades no académica en varones, y extraversión se asociaba con logro en pruebas de lenguaje y matemática principalmente en mujeres en primaria y sólo con logro en las pruebas de lenguaje en mujeres de secundaria,

Eysenck y Cookson (Nácher, 2002) encuentran que extraversión, asociada a estabilidad emocional favorece el rendimiento en pruebas de razonamiento verbal. Little, Lecci y Watkinson, (1992) confirman la relación negativa entre rendimiento y neuroticismo, argumentando que esto se debe a la falta de goce en lo personal, y sentido exagerado de la presión del tiempo que presentan los sujetos con este rasgo. 
Asimismo, Nácher evidencia una correlación significativa entre neuroticismo, propensión a la conducta antisocial y bajo rendimiento escolar. Los alumnos con un rendimiento escolar más bajo tenderían a ser más ansiosos, deprimidos, con sentimientos de culpa y baja autoestima, especialmente ante situaciones de evaluación.

Ampuero (1989) estudio la relación entre aspectos motivacionales de la personalidad (a través Inventario de Preferencias Personales de Edwards), rendimiento y elección vocacional. Dicha autora encuentra diferencias importantes en el patrón de relaciones tomando en cuenta la facultad: Arte aparece con niveles altos de rendimiento autónomo, persistencia, tolerancia al cambio y agresividad; Trabajo Social, con niveles altos de deferencia, orden y abatimiento, y bajos en exhibicionismo, dominancia y heterosexualidad; Derecho se encuentra puntajes altos en dominio y agresión; en Humanidades, mayor intrapercepción y necesidad de ser ayudado; y en Educación mayor afiliación y ayuda a otros.

No se puede excluir el rol de los factores contextuales, que resultan predictores del rendimiento al asociarse a los factores persona$\mathrm{les}^{5}$. El ambiente universitario resulta un espacio de descubrimiento intelectual, enriquecimiento personal, cuestionamiento y cambio que plantea nuevos retos, nuevos modelos, discernimientos y maneras de pensar (Papalia, 1992). El dominio de tareas, el sentimiento de competencia y valía personal se expresan en términos del rendimiento efectivo, aunque también el desenvolvimiento del alumno exitoso puede implicar menos conformismo y mayor ansiedad (Watson y Lindgren, 1991). El rendimiento inferior por el contrario, se asocia a sentimientos de inhibición, inseguridad, defensividad, inquietud, irresponsabilidad, actitudes negativas hacia el centro de estudios, oposición a la autoridad y ajuste emocional pobre.

$5 \quad$ Se entiende por variables personales a la percepción de los propios atributos de personalidad y el self ideal, y por contextuales, al soporte percibido de los padres, mejores amigos, profesores, grupo de pares y background familiar. 
Personalidad y rendimiento académico en estudiantes universitarios

\section{Objetivos}

Determinar la asociación entre características personales y rendimiento académico en universitarios.

Se plantearon los siguientes objetivos específicos:

- Determinar la correlación entre aspectos de personalidad y rendimiento académico en estudiantes universitarios de nuestro medio.

- Comprobar la validez y confiabilidad de instrumentos de evaluación de personalidad y rendimiento en estudiantes universitarios de nuestro medio.

- Comparar aspectos de personalidad y rendimiento académico en estudiantes universitarios de nuestro medio según sexo, edad, ciclo que cursan, autopercepción del rendimiento y fuentes de apoyo.

Se plantean hipótesis sobre el grado de correlación entre las variables rendimiento académico y personalidad.

\section{Metodología}

\section{Participantes}

Se trabajó con un total de 170 alumnos, en una proporción similar de hombres y mujeres (51.2 y $48.8 \%$, respectivamente) como se aprecia en la Figura 1. 


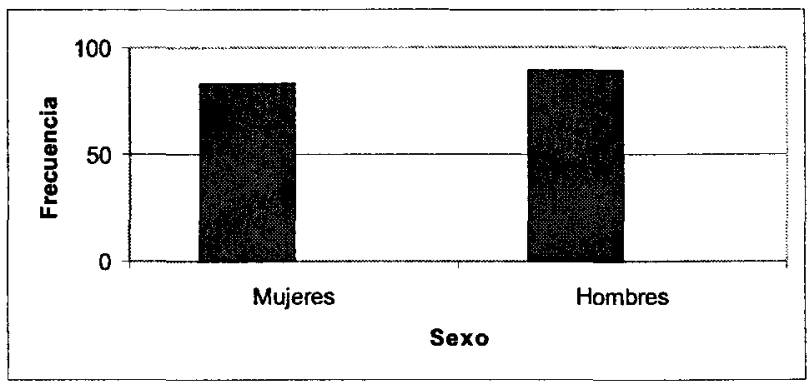

Figura 1. Participantes según sexo.

Predominan estudiantes entre 19 y 22 años, con un $56.5 \%$ del total (Figura 2), nacidos en Lima (78.2\%) (Figura 3), distribuidos equilibradamente por facultades, Ciencias $52.4 \%$ y Letras $47.6 \%$ (Figura 4).

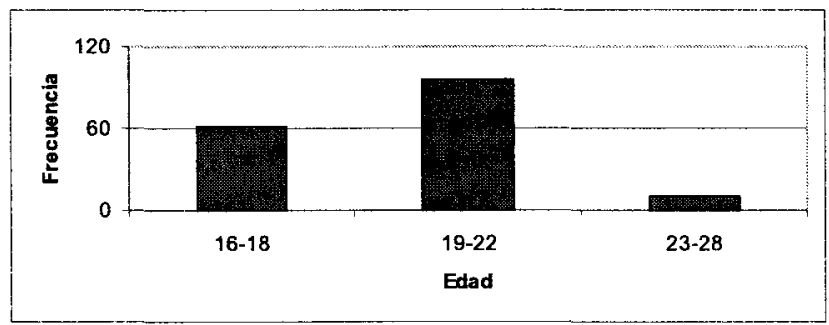

Figura 2. Participantes según edad.

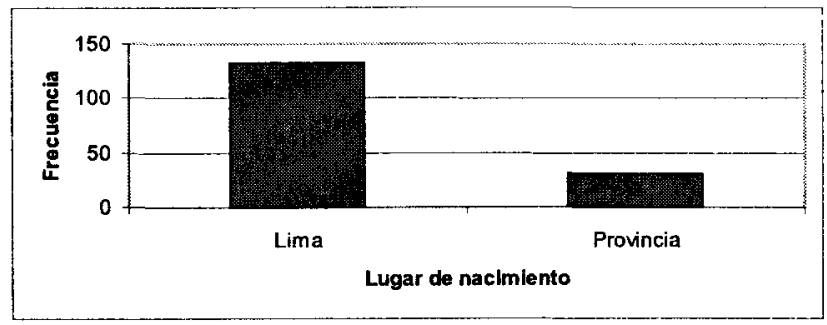

Figura 3. Participantes según lugar de nacimiento. 
Personalidad y rendimiento académico en estudiantes universitarios

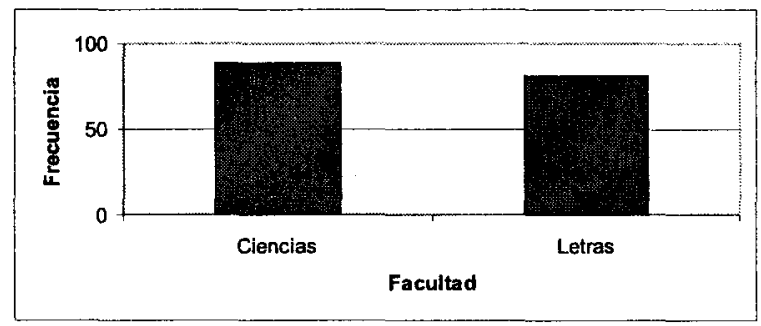

Figura 4. Participantes según facultad.

\section{Instrumentos}

El inventario de los Cinco Factores de Personalidad NEO PI-R (forma $\mathrm{S}$ ) de Costa y McCrae evalúa las cinco mayores dimensiones de la personalidad normal: extraversión, agradabilidad, conciencia, neuroticismo y apertura. Su forma autoadministrada (S) consta de 240 ítemes que se responden en una escala de cinco puntos. Costa y McCrae (1992) reportan un coeficiente alfa de confiabilidad de .56 a .81 en las escalas, así como la validez convergente, discriminante y de criterio externo del instrumento. Fue adaptado para universitarios peruanos por Cassaretto (1999) y mejorado por Niño de Guzmán, Calderón y Cassaretto (2001). El test distingue seis facetas y treinta rasgos de personalidad:

Neuroticismo (N): ansiedad (N1), hostilidad colérica (N2), depresión (N3), ansiedad social (N4), impulsividad (N5), vulnerabilidad (N6).

Extraversión (E): calidez (E1), gregarismo (E2), asertividad (E3), actividad (E4), búsqueda de sensaciones (E5), emociones positivas (E6).

Apertura (O): fantasía (O1), estética (O2), sentimientos (O3), acciones (O4), ideas (O5), valores (O6). 
Agradabilidad (A): confianza (A1), franqueza (A2), altruismo (A3), complacencia (A4), modestia (A5), benevolencia (A6).

Conciencia $(C)$ : competencia $(\mathrm{C} 1)$, orden $(\mathrm{C} 2)$, sentido del deber (C3), aspiraciones de logro (C4), autodisciplina (C5), reflexión (C6).

El inventario de Preferencias Personales de Edwards EPPS (1959) evalúa una compleja taxonomía de necesidades, entre ellas, la necesidad de logro (ACH), deferencia (DEF), orden (ORD), exhibicionismo (EXH), autonomía (AUT), afiliación (AFF), introspección (INT), necesidad de ayuda (SUC), dominancia (DOM), abatimiento (ABA), cuidado de otros (NUR), cambio (CHG), perseverancia (END), heterosexualidad (HET), agresión (AGG). Piedmont, McCrae y Costa examinaron la validez de las escalas de necesidad del inventario mediante la correlación con una medida de los cinco factores básicos de personalidad, el Inventario de Cinco Factores de Personalidad (NEO PI-R) en 330 estudiantes (223 mujeres y 107 hombres). Los resultados mostraron que tanto las versiones ipsativa como normativa del EPPS podían ser interpretadas significativamente con el modelo de los cinco factores, aunque el formato ipsativo del EPPS estándar tendía a disminuir los coeficientes (Piedmont, McCrae y Costa, 1992).

Ficha sociodemográgica. Con esta ficha se recogió información sobre: edad, ciclo académico, autopercepción del rendimiento, percepción de la propia motivación hacia el estudio y fuentes de apoyo.

Adicionalmente, se obtuvo el promedio ponderado estandarizado del participante, como medida de su rendimiento académico. Este promedio tiene la estructura de una puntuación estandarizada que permite comparaciones entre alumnos de diferentes cohortes y especialidades. 
Personalidad y rendimiento académico en estudiantes universitarios

\section{Procedimiento}

La investigación se llevó a cabo en dos etapas. En la primera se preparó a los evaluadores y se estableció contacto con los participantes. Se les explicó el propósito de la investigación y se les solicitó su consentimiento con la garantía de la confidencialidad. La aplicación de las pruebas fue colectiva. En la segunda etapa se hizo la devolución de resultados a los participantes y se registraron sus antecedentes académicos. Finalmente, los datos fueron procesados estadísticamente.

\section{Resultados}

El promedio estandarizado en la muestra resultó 50.3 con una desviación estándar 5.5 que no difiere mayormente de lo esperado, esto es, la muestra puede considerarse representativa de la población.

En término de la autopercepción del rendimiento es considerado por la mayoría como regular (74\%), mientras que sólo un $12.4 \%$ lo considera bueno. Adicionalmente (Figura 6), el nivel de motivación que mantienen es solo mediano y muchos (14\%) confiesan tener una baja motivación.

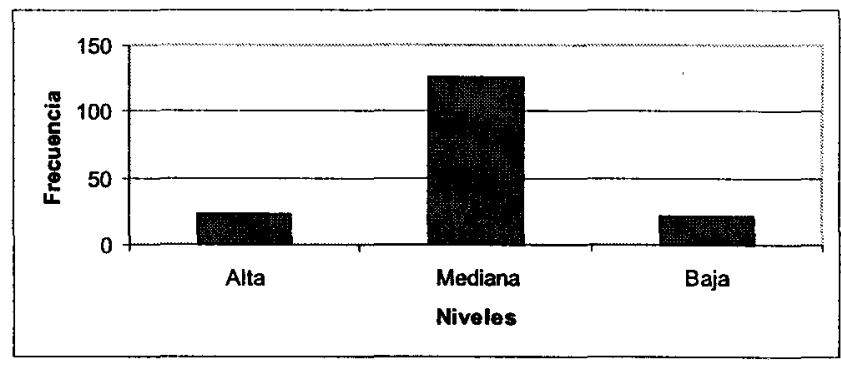

Figura 6. Percepción de motivación. 
La mayoría se encuentra matriculada en un ciclo regular, llevando entre 18.5 y 23.5 créditos $(60.7 \%)$. Resulta importante mencionar que la principal fuente de apoyo percibida por la mayoría son los padres y que el apoyo de la universidad y de los profesores son percibidos como mínimos (Figura 7).

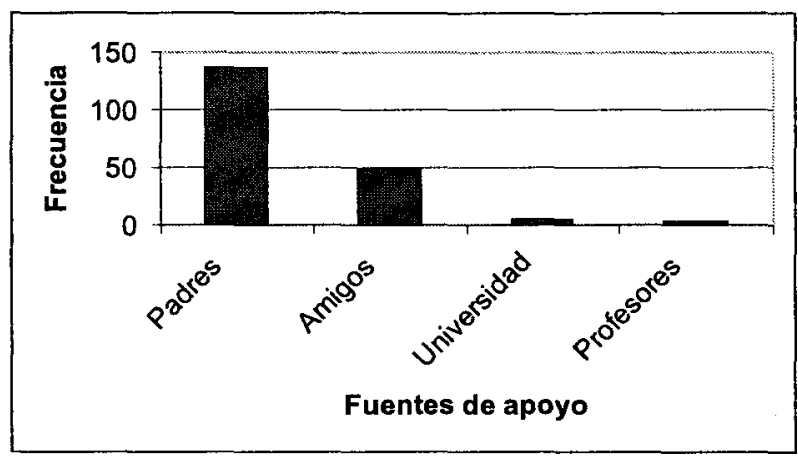

Figura 7. Percepción de fuentes de apoyo.

Con respecto a la confiabilidad, se logró determinar el nivel alto de que ofrecen ambos instrumentos, tal como se venía ya trabajando en investigaciones previas (Niño de Guzmán, Calderón y Cassaretto, 2001).

En cuanto a la confiabilidad de los instrumentos de personalidad, se logró comprobar el alto nivel que ofrecen ambos, tal como se había ya visualizado en investigaciones previas (Niño de Guzmán, Calderón y Cassaretto, 2001). En el caso del EPPS (Ampuero, 1989; Checa, 1989; Edwards, 1959), se aplicó la fórmula de SpearmanBrown, basada en el método de correlación por mitades, encontrándose en todos los casos correlaciones significativas $(p<.05)$ y buenos coeficientes de confiabilidad para todas las escalas (Cuadro 1). 
Personalidad y rendimiento académico en estudiantes universitarios

\section{Cuadro 1}

Confiabilidad del EPPS mediante coeficiente Spearman-Brown

\begin{tabular}{|llc|}
\hline Escala & $r$ & Confiabilidad \\
\hline ACH & .42 & .59 \\
DEF & .35 & .52 \\
ORD & .56 & .72 \\
EXH & .40 & .57 \\
AUT & .50 & .67 \\
AFF & .51 & .67 \\
INT & .48 & .65 \\
SUC & .63 & .77 \\
DOM & .64 & .78 \\
ABA & .74 & .85 \\
NUR & .56 & .72 \\
CHG & .65 & .79 \\
END & .61 & .76 \\
HET & .68 & .81 \\
AGG & .64 & .78 \\
\hline
\end{tabular}

En relación con el NEO PI-R, la validez de constructo fue verificada mediante el análisis de su estructura factorial, determinándose la existencia de cinco factores que coinciden con los del modelo original. La confiabilidad del NEO PI-R, se determinó mediante correlaciones ítem-test y alfa de Cronbach, como se puede observar en el Cuadro 2, en el que aparecen todas las dimensiones y facetas con puntajes de confiabilidad significativos.

\section{Cuadro 2}

Confiabilidad del NEO-PI

\begin{tabular}{|cccccccccc|}
\hline Faceta & Alfa & Faceta & Alfa & Faceta & Alfa & Faceta & Alfa & Faceta & Alfa \\
\hline N & .90 & E & .91 & O & .85 & A & .84 & C & .91 \\
N1 & .72 & E1 & .68 & O1 & .64 & A1 & .74 & C1 & .72 \\
N2 & .78 & E2 & .77 & O2 & .71 & A2 & .71 & C2 & .68 \\
N3 & .74 & E3 & .79 & O3 & .65 & A3 & .70 & C3 & .54 \\
N4 & .70 & E4 & .67 & O4 & .64 & A4 & .65 & C4 & .80 \\
N5 & .60 & E5 & .60 & O5 & .78 & A5 & .67 & C5 & .75 \\
N6 & .70 & E6 & .81 & O6 & .41 & A6 & .50 & C6 & .71 \\
\hline
\end{tabular}

$p<.05$ 
Los instrumentos de personalidad usados no miden exactamente los mismos aspectos de los rasgos básicos; así, mientras que las escalas del NEO PI-R se concentran en aspectos estructurales de la personalidad, las del EPPS se refieren a aspectos motivacionales de la misma. En este sentido, y dado que luego esta relación mostrará importancia al asociar rendimiento con personalidad, resultó conveniente estudiar la concurrencia (vía correlaciones) entre las escalas de los dos instrumentos, tal como se aprecia en el Cuadro 3. Se puede observar la presencia de correlaciones significativas entre las escalas del EPPS y las dimensiones del NEO PI-R. Cabe resaltar que las mayores correlaciones positivas se presentan entre neuroticismo y abatimiento, extraversión y dominancia, agradabilidad con cuidado a otros, abatimiento y afiliación, y conciencia con perseverancia y dominancia. Las mayores correlaciones negativas se presentan entre neuroticismo y dominancia, extraversión con orden, perseverancia y abatimiento, apertura con orden, y agradabilidad con agresión y autonomía.

\section{Cuadro 3}

Correlaciones EPPS- NEO PI-R

\begin{tabular}{|c|c|c|c|c|c|}
\hline \multicolumn{6}{|c|}{ NEO PI R } \\
\hline EPPS & $\mathrm{N}$ & $\mathrm{E}$ & $\mathrm{O}$ & $\mathrm{A}$ & $\mathrm{C}$ \\
\hline $\mathrm{ACH}$ & -.17 & & & -.17 & .19 \\
\hline DEF & & -.18 & & .16 & \\
\hline ORD & & -.38 & $-0,42$ & & .29 \\
\hline EXH & & .23 & -.16 & -.23 & \\
\hline AUT & & & & -.30 & -.21 \\
\hline $\mathrm{AFF}$ & & .29 & .17 & .33 & -.18 \\
\hline INT & & & .21 & & \\
\hline SUC & .22 & & & .26 & -.18 \\
\hline DOM & -.33 & .34 & & -.25 & .30 \\
\hline $\mathrm{ABA}$ & .41 & -.33 & -.16 & .35 & \\
\hline NUR & & .15 & & .41 & \\
\hline CHG & & .24 & .23 & & \\
\hline END & & -34 & -.27 & & .36 \\
\hline AGG & & & & -.43 & -.20 \\
\hline
\end{tabular}

$p<.05$ 
Personalidad y rendimiento académico en estudiantes universitarios

En el Cuadro 4 aparece la conformación de las escalas y facetas del NEO PI-R. apertura obtiene la media más alta, seguida de conciencia. En caso del EPPS, no consignamos estadísticos, dado que la prueba convierte los puntajes brutos de todas las escalas a puntuaciones $T$ de media 50 y desviación estándar 10 .

\section{Cuadro 4}

Escalas y Facetas del NEOPI-R

\begin{tabular}{|lcc|}
\hline \multicolumn{1}{|c|}{ Escala del NEO PI-R } & $M$ & $D E$ \\
\hline N : Neuroticismo & 95.1 & 21.5 \\
E : Extroversión & 110.9 & 22.2 \\
O : Apertura & 114.4 & 17.3 \\
A : Agradabilidad & 108 & 16.7 \\
C : Conciencia & 111.7 & 21.2 \\
\hline FN1 : Ansiedad & 17.9 & 4.9 \\
FN2 : Ansiedad colérica & 13.7 & 4.7 \\
FN3 : Depresión & 16 & 5.4 \\
FN4 : Ansiedad social & 15.7 & 5.1 \\
FN5 : Impulsividad & 17.8 & 4.3 \\
FN6 : Vulnerabilidad & 14 & 5 \\
\hline FE1 : Calidez & 21.6 & 4.3 \\
FE2 : Gregarismo & 18.4 & 5.8 \\
FE3 : Asertividad & 16 & 5.5 \\
FE4 : Actividad & 15.7 & 4.1 \\
FE5 : Búsqueda de sensaciones & 19 & 4.7 \\
FE6 : Emociones positivas & 20.3 & 5.8 \\
\hline FO1 : Fantasía & 20.5 & 4.4 \\
FO2 : Estética & 19 & 5.3 \\
FO3 : Sentimientos & 21.1 & 4.3 \\
FO4 : Acciones & 16.8 & 4.2 \\
FO5 : Ideas & 18.7 & 5.4 \\
FO6 : Valores & 18.3 & 3.4 \\
\hline FA1 : Confianza & 16.6 & 4.9 \\
FA2 : Franqueza & 17.4 & 5.2 \\
FA3 : Altruismo & 22.1 & 4.2 \\
FA4 : Complacencia & 15.5 & 4.8 \\
FA5 : Modestia & 15.7 & 4.5 \\
FA6 : Benevolencia & 20.8 & 2.8 \\
\hline FC1 : Competencia & 19.4 & 4.2 \\
FC2 : Orden & 17.3 & 4.8 \\
FC3 : Sentido del deber & 21.4 & 4 \\
FC4 : Aspiraciones de logro & 20 & 5.1 \\
FC5 : Autodisciplina & 16.1 & 4.8 \\
FC6 : Reflexión & 17.4 & 4.9 \\
\hline
\end{tabular}

$N=170$ 
Antes de analizar la relación entre las distintas variables con el rendimiento, se evaluó las diferencias en las escalas de personalidad según sexo, encontrándose diferencias significativas en las escalas mencionadas en el Cuadro 5. Sin embargo, como se verá luego, estas diferencias no llegan a tener efecto relevante en el rendimiento.

\section{Cuadro 5}

Diferencias en escalas de personalidad según sexo

\begin{tabular}{|lcccc|}
\hline & \multicolumn{2}{c}{ Femenino } & \multicolumn{2}{c|}{ Masculino } \\
\hline Escala & $M$ & $D E$ & $M$ & $D E$ \\
$\mathrm{~N}$ & 100.3 & 21.6 & 90.1 & 20.4 \\
$\mathrm{O}$ & 117.5 & 18.8 & 111.4 & 15.3 \\
$\mathrm{~N} 1$ & 19.1 & 4.9 & 16.8 & 4.6 \\
$\mathrm{~N} 3$ & 17.2 & 5.4 & 14.8 & 5.2 \\
$\mathrm{~N} 6$ & 15.9 & 4.8 & 12.2 & 4.5 \\
$\mathrm{E} 1$ & 22.6 & 4.5 & 20.6 & 3.9 \\
E2 & 19.6 & 5.7 & 17.2 & 5.6 \\
O2 & 20.6 & 4.6 & 17.5 & 5.5 \\
O3 & 22.2 & 4.3 & 20.1 & 4.2 \\
O4 & 17.7 & 4.3 & 16.1 & 4.0 \\
O5 & 17.7 & 5.6 & 19.6 & 5.0 \\
ORD & 48.3 & 10.1 & 51.6 & 9.7 \\
AFF & 53.2 & 9.2 & 46.9 & 9.8 \\
CHG & 52.7 & 9.9 & 47.4 & 9.5 \\
END & 48.4 & 10.4 & 51.5 & 9.5 \\
HET & 46.9 & 10.4 & 53.0 & 8.7 \\
\hline
\end{tabular}

$p<.01$

$\mathrm{Al}$ analizar la relación entre las variables representadas por el NEO PI-R y el rendimiento académico, la única correlación significativa aparece con el factor conciencia (Cuadro 6), lo cual coincide con la teoría de referencia. 
Personalidad y rendimiento académico en estudiantes universitarios

\section{Cuadro 6}

Correlación NEO PI-R y rendimiento

\begin{tabular}{|cccccc|}
\hline Dimensión & $\mathrm{N}$ & $\mathrm{E}$ & $\mathrm{O}$ & $\mathrm{A}$ & $\mathrm{C}$ \\
\hline$r$ & -.109 & -.053 & .011 & -.001 & $.330^{*}$ \\
\hline
\end{tabular}

$* p<.05$

Tal como se aprecia en el Cuadro 7 se encuentran correlaciones significativas entre rendimiento académico y casi todas las facetas que conforman el factor conciencia: aspiración de logro (C4), autodisciplina (C5), competencia (C1), reflexión (C6), y sentido del deber (C3). Las correlaciones que aparecen con la faceta ideas (O5) y con la faceta impulsividad (N5) no han sido reportadas anteriormente en la literatura y llaman la atención.

\section{Cuadro 7}

Correlación facetas NEOPI-R y rendimiento

\begin{tabular}{|cccccccc|}
\hline Faceta & $\mathrm{C} 4$ & $\mathrm{C} 5$ & $\mathrm{Cl}$ & $\mathrm{C} 6$ & $\mathrm{C} 3$ & $\mathrm{O} 5$ & $\mathrm{~N} 5$ \\
\hline$r$ & 0.34 & 0.31 & 0.27 & 0.26 & 0.25 & 0.16 & -0.16 \\
\hline
\end{tabular}

$p<.05$

En el caso del EPPS (Cuadro 8), se aprecian correlaciones significativas entre rendimiento y sólo tres escalas: perseverancia (END), cambio (CHG) y agresión (AGG).

Cuadro 8

Correlación EPPS y rendimiento

\begin{tabular}{|cccc|}
\hline Escala & END & CHG & AGG \\
\hline$r$ & .27 & -.20 & -.16 \\
\hline
\end{tabular}

$p<.05$ 
$\mathrm{Al}$ analizar las correlaciones entre el rendimiento estandarizado y otras variables independientes (Cuadro), sólo destacan cuatro de ellas: autopercepción del rendimiento, edad, percepción de la motivación para el estudio y apoyo de la universidad (otras variables como sexo y apoyo de padres no muestran correlación significativa). Cabe mencionar, sin embargo, que estas variables, salvo la edad, muestran correlaciones con alguna de las variables de personalidad, siendo remarcables las correlaciones de percepción de la motivación con aspiración de logro (C4) ( $r=.51)$ y autodisciplina (C6) $(r=.36)$, así como la correlación entre percepción de rendimiento y aspiración de logro $(r=.34)$, todas significativas al nivel $p=.05$. Estas relaciones con personalidad, como se verá adelante, servirán para matizar las correlaciones con rendimiento.

\section{Cuadro 9}

Correlación rendimiento con otras variables independientes

\begin{tabular}{|ccccc|}
\hline & $\begin{array}{c}\text { Percepción de } \\
\text { Rendimiento }\end{array}$ & Edad & $\begin{array}{c}\text { Percepción de la } \\
\text { motivación para el } \\
\text { estudio }\end{array}$ & $\begin{array}{c}\text { Percepción de } \\
\text { apoyo de la } \\
\text { universidad }\end{array}$ \\
\hline$r$ & .41 & -.36 & .30 & .21 \\
\hline
\end{tabular}

$p<.05$, dos colas.

Para lograr un nivel de mayor profundidad en la comprensión de los hallazgos, se realizó un análisis integrado de los resultados anteriores, mediante un análisis de regresión múltiple tomando como variable dependiente el rendimiento (promedio estandarizado) y según el método forward, dada la presencia de correlaciones entre las variables independientes del modelo y la consiguiente necesidad de controlar redundancias. Se ingresaron las variables que correlacionaban con rendimiento, en el siguiente orden: primero las facetas del NEO PI-R, luego las escalas del EPPS y finalmente las variables de la ficha sociodemográfica. 
El contraste global $F$ de la regresión resultó significativo al $1 \%$ $(F(5.159)=16.52)$, con un $r$ cuadrado de .342 y un $r$ ajustado .321 , y luego de realizar los contrastes $t$ individuales para determinar la significación de cada variable independiente, se obtuvo el conjunto de variables explicativas que se muestran en el Cuadro 10, junto con los coeficientes Beta que miden su importancia relativa.

\section{Cuadro 10}

Análisis de Regresión Múltiple: Variables de investigación y rendimiento

\begin{tabular}{|lcccc|}
\hline & $B$ & Beta & $t$ & Sig. \\
\hline C4 & 0.18 & 0.16 & 2.20 & .03 \\
C6 & 0.17 & 0.15 & 2.09 & .04 \\
CHG & -0.07 & -0.13 & -1.99 & .04 \\
Edad & -0.89 & -0.31 & -4.83 & .00 \\
Percepción de rendimiento & 2.77 & 0.25 & 3.58 & .00 \\
\hline
\end{tabular}

\section{Discusión}

El tema central de este estudio es la relación entre el rendimiento académico y las características de personalidad en alumnos de primeros años de las facultades de Ciencias y Letras de una universidad privada. Consideramos que el conocimiento de esta relación resulta importante no sólo como objeto de investigación sino también para la planificación en una institución con fines académicos en constante proceso de mejora.

Los datos indican que se ha accedido a un grupo bastante homogéneo en cuanto a una serie de características: porcentaje similar de hombres y mujeres, la mayor parte de la capital, en proporción semejante de las facultades de Ciencias y Letras.

Es un hallazgo interesante verificar la estructura básica de personalidad del modelo de los cinco factores a través de los resultados NEO PI-R, lo cual replica lo obtenido en otros estudios en el Perú 
(Cassaretto, 1999, Niño de Guzmán et al., 2001, Astonitas, 2002). De forma similar se confirma la confiabilidad de las escalas del EPPS. Asimismo, las correlaciones halladas entre ambas pruebas coinciden con las obtenidas por Piedmond, McCrae y Costa (1992).

En relación a lo obtenido por los factores y facetas de personalidad se encontró que este grupo de estudiantes puntuaban alto en la dimensión apertura $(\mathrm{O})$, conciencia, extraversión $(\mathrm{E})$, y más bajo en neuroticismo $(\mathrm{N})$. Lo cual nos permite describir a esta muestra de estudiantes como receptivos ante lo nuevo, con mayor curiosidad e interés por lo que los rodea, con tendencia a persistir en tareas que son un reto y en las que podría ser aplicado un patrón de excelencia. Al encontrarse entre la etapa de la adolescencia tardía y adultez temprana se orientan hacia el grupo social que los rodean, sus principales fuentes de interés estarían en el establecimiento de vínculos interpersonales de mayor intimidad a través de los amigos y la pareja, y con mayor preocupación por el bienestar común y los ideales del grupo evidenciando que nos encontramos ante un grupo de jóvenes mental y emocionalmente saludables.

Los resultados señalan la fuerte relación entre el rasgo de personalidad conciencia y el rendimiento académico, lo cual responde a la propia definición del rasgo que alude a características como persistencia, competencia y motivación en la conducta y en las metas. Esto coincide con lo encontrado en otras investigaciones anteriormente citadas.

El análisis a nivel de facetas nos permite concluir que los sujetos con alta conciencia presentan mayor rendimiento al ser este sustentado en altas aspiraciones de logro (C4), mayor capacidad reflexiva (C6), disciplina personal (C5), sentido de competencia (C1) y del deber (C3). Esto nos da un posible perfil de un "buen estudiante universitario" quien se caracterizaría como altamente motivado por sus metas, con confianza en sus habilidades, con capacidad para planifi- 
Personalidad y rendimiento académico en estudiantes universitarios

car de forma cuidadosa y prudente sus acciones, y comprometido ante las obligaciones académicas y personales en las que se involucra.

Las correlaciones con la faceta ideas (O5) e impulsividad (N5), si bien novedosas, no se contradicen con lo mencionado en el párrafo anterior debido a que se sabe que los sujetos con baja impulsividad son capaces de tolerar las frustraciones académicas de mejor forma y pueden perseverar en sus intentos de logro. La apertura a las ideas, por otro lado, está fuertemente asociada con una actitud curiosa intelectualmente y con un foco amplio de intereses que favorece una disposición del estudiante al estudio.

Las relaciones entre rendimiento y aspectos motivacionales de la personalidad se centran alrededor de las necesidades de perseverancia (END) positivamente y, cambio (CHG) y agresión (AGG) negativamente. En el caso de perseverancia se encuentra armonía con los resultados señalados en conciencia. Se detectó que ambas escalas correlacionan fuertemente. Esto nos haría sospechar que miden un factor común. Por otro lado, cambio está asociado con el deseo de realizar actividades y rutinas diferentes, y con el deseo de conocer lugares nuevos, lo que se contrapone con el relativo sedentarismo del estudiante universitario peruano, cuyo aprendizaje depende mayormente de su permanencia y dedicación casi exclusiva a la universidad, más que de nutrirse de nuevas experiencias que enriquezcan su aprendizaje. Por eso, sería relevante analizar el efecto en el rendimiento, de la práctica de actividades de tipo laboral, social, recreativa e intelectual, y confrontarlas con otras estrategias de aprendizaje más activas para el alumno. La agresión está definida como la actitud de criticar a otros públicamente, hacer burla de los demás, tomar venganza; todas actitudes que harían a un joven más difícil integrarse a un medio como el universitario, en donde el trabajo en grupo y el intercambio de ideas es importante. Sin embargo, algunas otras características de la agresión, tal como está entendida en el EPPS, se refieren a la habilidad de decir a otros lo que se piensa de ellos o atacar 
el punto de vista contrario y suelen relacionarse con elementos de asertividad. La escala del NEO PI-R más relacionada con asertividad (E3) no muestra correlación significativa con rendimiento e indicaría que son los aspectos negativos de agresión los que predominan en la correlación inversa entre rendimiento y esta escala.

Se encontraron correlaciones positivas entre el autopercepción del rendimiento, percepción de la motivación para el estudio, y rendimiento. Sin embargo, cabe resaltar que no fue objetivo de este estudio evaluar las dos primeras variables mencionadas sino mantenerlas como control y aunque llama la atención la importancia que dichas variables cobraron en las correlaciones, en esta investigación solo podrían ser objeto de especulación. Queda ampliar nuevas líneas de trabajo que profundicen en el tema, a través de instrumentos específicamente diseñados para ello.

Las otras variables de control que mostraron relación con rendimiento fueron edad y percepción de apoyo por parte de la universi$d a d$. La relación negativa de la edad con el rendimiento se explica por que el estudio se circunscribe a alumnos de los dos primeros años de estudios y en este caso, alumnos de mayores edades suelen estar retrasados. En cambio, la correlación de percepción de apoyo y rendimiento es positiva. Sin embargo resulta importante mencionar que estos estudiantes perciben como principal fuente de apoyo a sus padres, más que a la universidad o sus profesores. Estos datos coinciden con algunos estudios que señalan que, a diferencia de lo que mucha gente opina respecto a los niveles de conflicto que se presentan entre jóvenes y sus padres, son éstos a quienes se recurre cuando se trata de decisiones importantes Podemos poner como ejemplo el terreno académico y vocacional (Papalia, 1992).

De las 14 variables que correlacionaron significativamente con rendimiento, el análisis de regresión múltiple permite considerar a cinco de ellas como las de mayor poder explicativo. Tres de ellas son 
de personalidad (C4, C6 y $\mathrm{CHG})$. Aspiraciones de logro y reflexión (en sentido directo) y cambio (en sentido inverso) las que determinan parte del rendimiento académico ya que estas incorporan los otros atributos señalados. Así se puede considerar que tener metas claras, altas y realistas prudentemente evaluadas junto con una mayor disposición a focalizarse en el ambiente universitario redunda en un mejor desempeño.

De las cuatro variables sociodemográficas que correlacionan con rendimiento: percepción de rendimiento, percepción de motivación hacia el estudio, apoyo percibido de la universidad y edad, las tres primeras también muestran correlaciones con personalidad y en particular percepción de motivación tiene mucha mayor correlación con personalidad, y podría interpretarse más como una variable cuyo efecto se integra a las anteriores, a diferencia de percepción de rendimiento que mantiene un cierto grado de independencia. Los resultados del análisis de regresión muestran que percepción de rendimiento queda como una de las variables finales importantes en el modelo, adicionalmente a las de personalidad y con poder explicativo propio. Aunque la Edad también resulta variable explicativa de importancia, esto se explica por los mismos motivos mencionados al tratar las correlaciones.

\section{Referencias}

Astonitas, M. (2002). Característica de personalidad y riesgo de adicción a Internet en un grupo de estudiantes universitarios usuarios regulares de Internet. Tesis para optar el título de Licenciada en Psicología, Pontificia Universidad Católica del Perú, Lima.

Ampuero, G. (1989). El Inventario de Preferencias Personales de Edwards en estudiantes de la PUCP. Manuscrito no publicado. 
Barrick, M. R. y Mount, M. K. (1991). The Big Five personality dimensions and job performance: A meta-analysis. Personnel Pschology,44, 1-26.

Cassaretto, M. (1999). Adaptación del inventario de Personalidad $N E O P I-R$. Tesis para optar el título de Licenciada en Psicología, Pontificia Universidad Católica del Perú, Lima.

Cascón, I. (2002). Predictores del rendimiento académico en alumnos de primero y segundo de BUP (en línea), usal.es.inico/ investigación/jornadas/jornada2 /comun/c19.html.

Claux, M.L. (1998). School performance in Peruvian adolescents: A psychological perspective on person and context predictors. Tesis para optar el grado de Doctor, Katholieke Universiteit Nijmegen. Nimega, Holanda: Mediagroep KUN/AZN.

Costa, P. T., Jr. y McCrae, R. R. (1992c). Revised NEO Personality Inventory (NEO PI-R) and NEO Five-Factor Inventory (NEOFFI) Professional Manual. Odessa, Florida: Psychological Assessment Resources.

Checa, J. (1989). Estudio de confiabilidad y validez de la investigación autodirigida de Holland (Forma E) Test de Intereses Vocacionales. Tesis para optar el título de licenciado en psicología, Pontificia Universidad Católica del Perú, Lima.

Digman, J. (1989). Five robust trait dimensions: development, stability and utility. Journal of Personality and Social Psychology, 57(2), 196-214.

Digman, J. (1990). Personality structure: emergence of the Five-Factor Model. Annual Review of Psychology, 41, 417-440.

Edwards, A. (1959). Edwards Personal Preference Schedule (Revised manual). Nueva York: The Psychological Corporation.

Gelatly, I. A. (1996). Concientiousness and task performance: test of a cognitive process model. Journal of Applied Psychology, 81(5), 474-482.

Graziano, W. G. y Ward, D. (1992). Probing the Big Five in adolescence: Personalitiy and adjustment during a developmental transition. Journal of Personality, 60(2), 426-439. 
Personalidad y rendimiento académico en estudiantes universitarios

Hogan, R., Johnson, J. y Briggs, J. (1997). Handbook of personality psychology. San Diego: Academic Press.

Little B. R., Lecci, L. y Watkinson, B. (1992). Personalitiy and personal projects: Linking Big Five and PAC units of analysis. Journal of Personality, 60(2), 501-525.

McCrae, R. (1994). Openess to experience as basic dimension of personality. Imagination, Cognition and Personality, 13, 39-55.

McCrae, R. R. y Costa, P. T., Jr. (1997). Personality Trait Structure as Human Universal. American Psychologist, 52, 509-516.

Nácher, V. (2002). Personalidad y rendimiento académico. Jornades de Foment de la Investigación. Universitat Jaume. En línea: google.com/search? q=personalidad $+\mathrm{y}$ rendimiento académico.

Niño de Guzmán, I., Calderón, A., Escalante, M., Lira, T., Morote, R. y Ruda, L. (2000). Personalidad ansiedad estado rasgo e ingreso a la universidad en alumnos preuniversitarios. Revista de Psicología PUCP, 18(2), 341-372.

Niño de Guzmán, I., Calderón, A. y Cassaretto, M. (2001). Rasgos de personalidad y rendimiento académico en estudiantes universitarios de la PUCP. Primera etapa. Lima: Dirección Académica de Investigación PUCP.

Papalia, D. (1992). Desarrollo Humano. México: McGraw Hill.

Piedmont, R. L., McCrae, R. R. y Costa, P. T., Jr. (1992). An assessment of the Edwards Personal Preference Schedule from the perspective of the Five-Factor Model. Journal of Personality Assessment. 58 (1), 67-78.

Watson, R. y Lindgren, H. (1992). Psicología del niño y del adolescente. México: Limusa. 